\title{
Visuelle Abstracts für Evidenzsynthesen
}

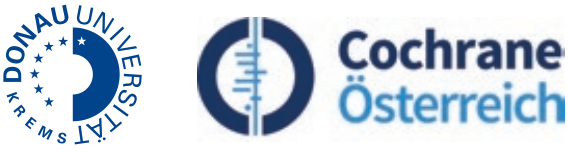

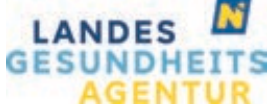

ebminfo.at
NOE GUS

ebninfo.at
D ie Donau-Universität Krems unterhält zwei evidenzbasierte Informationszentren für Pflegende und für Ärztinnen und Ärzte der niederösterreichischen Kliniken: ebninfo.at und ebminfo.at. Dort werden auf Anfrage Rapid Reviews erstellt, welche in kurzer Zeit Studien zu bestimmten Themen zusammenfassen. Das Lesen dieser Evidenzsynthesen kann aber aufgrund der Fachbegriffe und vieler Zahlen für ungeübte Leser wissenschaftlicher Literatur herausfordernd sein. Wir haben daher ein grafisches Darstellungsformat entwickelt, um die Ergebnisse der Studien möglichst verständlich, anschaulich und klar darstellen zu können.

Solche visuellen Abstracts werden zunehmend beliebter, um Studienergebnisse zusätzlich zum Text zu präsentieren. Dabei handelt es sich um grafische Zusammenfassungen der wichtigsten Informationen, die normalerweise im schriftlichen Abstract eines Artikels dargestellt werden. Ziel ist die 2-dimensionale grafische Darstellung des Ergebnisses mit der Richtung des Effekts und dem Vertrauen in das Ergebnis.

Der Aufbau unserer visuellen Abstracts ist in der Regel immer gleich gegliedert und soll den Lesern rasch vermitteln, ob die dargestellten Studienergebnisse relevant sind:

- Definition der Untersuchungsgruppe

- zu vergleichende Interventionen oder Risikofaktoren

- Darstellung der Ergebnisse je Endpunkt

abbildung des Vertrauens in die Evidenz

Interpretation der Ergebnisse

Bilder, Farben und Symbole werden zur Verdeutlichung der Ergebnisse angewendet. Dementsprechend werden statistisch und klinisch relevante Ergebnisse je Studienendpunkt entsprechend farblich gekennzeichnet sowie symbolisch darge-

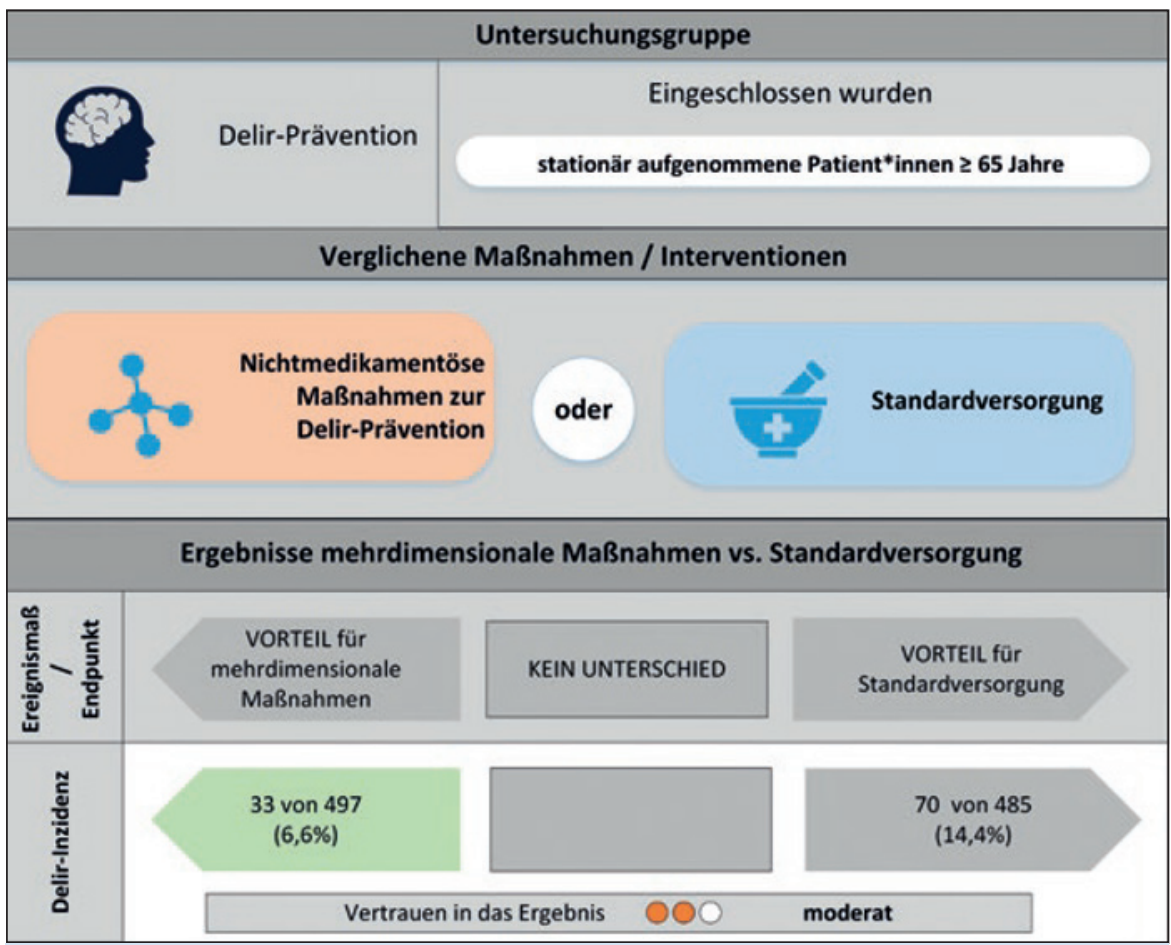

Auszug aus einem visuellen Abstract über Maßnahmen zur Delir Prävention im Krankenhaus: https://ebninfo.at/vorbeugung-eines-delirs-im-kh

stellt. Ergänzt werden diese Darstellungen durch kurze Texte und die entsprechenden Zahlen. Die grafischen Elemente sollen die jeweiligen Studienergebnisse verdeutlichen und somit zur Verständlichkeit, Übersichtlichkeit und raschen Vermittlung der Forschungsergebnisse beitragen.

Wir erstellen seit rund einem Jahr visuelle Abstracts für unsere Rapid Reviews und erhalten viele positive Rückmeldungen unserer Leserinnen und Leser. Das bestärkt unser Bestreben, Forschungsergebnisse nicht nur wissenschaftlich korrekt wiederzugeben, sondern auch die Darstellung eindeutiger und besser verständlich zu gestalten.
Martin Fangmeyer, $\mathrm{BScN}, \mathrm{MScN}$

Evidenzbasiertes Informationszentrum für Pflegende - Donau-Universität Krems Department für Evidenzbasierte Medizin und Evaluation - Cochrane Zentrum Österreich martin.fangmeyer@donau-uni.ac.at www.ebninfo.at | \#ebninfoAT

Dr. ${ }^{\text {in }}$ Anna Glechner

Evidenzbasiertes Informationszentrum für ÄrztInnen - Donau-Universität Krems Department für Evidenzbasierte Medizin und Evaluation - Cochrane Zentrum Österreich anna.glechner@donau-uni.ac.at www.ebminfo.at | \#ebminfoAT 